\title{
Mechanical ventilation and histological fibrosis in patients with acute respiratory distress syndrome undergoing open lung biopsy
}

Hsin-Hsien Li

Chang Gung University

Chih-Wei Wang

Chang Gung Memorial Hospital

Chih-Hao Chang

Chang Gung Memorial Hospital

Chung-Chi Huang

Chang Gung Memorial Hospital

Han-Shui Hsu

Taipei Veterans General Hospital

Li-Chung Chiu (D pomd54@cgmh.org.tw)

Chang Gung Memorial Hospital

\section{Research Article}

Keywords: Mechanical ventilation, Acute respiratory distress syndrome, Open lung biopsy, Histology, Diffuse alveolar damage, Pulmonary fibrosis, Idiopathic pulmonary fibrosis, Outcomes

Posted Date: January 21st, 2022

DOI: https://doi.org/10.21203/rs.3.rs-1277185/v1

License: (9) This work is licensed under a Creative Commons Attribution 4.0 International License. Read Full License 


\section{Abstract}

Background: Mechanical ventilation is the most important support treatment for acute respiratory distress syndrome (ARDS); however, it brings with it the risk of ventilator-induced lung injury, which can lead to pulmonary fibrosis and prolonged mechanical ventilation. Our objective in this study was to examine the correlation between ventilator settings and histological fibrosis in ARDS patients. We also examined the impact of histological fibrosis on clinical outcomes and mortality. Methods: We performed a retrospective analysis of patients with ARDS who received open lung biopsy in a tertiary care referral center in Taiwan between March 2006 and December 2019. Mechanical ventilator settings were recorded at the time of ARDS diagnosis and on the day of biopsy. Results: A total of 68 ARDS patients who had received open lung biopsy with diffuse alveolar damage (DAD; the hallmark pathology of ARDS) were analyzed and stratified into non-fibrosis $(n=56)$ and fibrosis groups $(n=12)$. Mechanical power and airway pressures at the time of ARDS diagnosis and airway pressures on the day of biopsy were significantly higher in the fibrosis group than in the non-fibrosis group, and lung compliance on the day of biopsy was significantly lower. The duration of ventilator usage and time spent in the intensive care unit and hospital stay were all significantly higher in the fibrosis group. Hospital mortality was higher in the fibrosis than in the non-fibrosis group ( $67 \%$ vs. $57 \%, p=0.748)$. In the fibrosis group, patients with AE-IPF $(n=5)$ received higher ventilator load and faced higher hospital mortality than DAD with fibrotic phase ( $n$ $=7)(80 \%$ vs. $57 \%, p=0.242)$. A multivariable logistic regression model demonstrated that mechanical power at ARDS diagnosis and ARDS duration before biopsy were independently associated with histological fibrosis at open lung biopsy (odds ratio 1.493 [95\% $\mathrm{Cl} 1.014-2.200], \mathrm{p}=0.042$; odds ratio 1.160 [95\% Cl 1.052-1.278], $p=0.003$, respectively). Conclusions: Our findings indicate that prompt action aimed at staving off injurious mechanical stretching of lung parenchyma and subsequent progression to fibrosis may have a positive effect on clinical outcomes. Keywords: Mechanical ventilation, Acute respiratory distress syndrome, Open lung biopsy, Histology, Diffuse alveolar damage, Pulmonary fibrosis, Idiopathic pulmonary fibrosis, Outcomes

\section{Background}

Acute respiratory distress syndrome (ARDS) is a heterogeneous syndrome with complex pathophysiologic mechanisms characterized by severe hypoxemia and high mortality [1]. The pathogenesis of ARDS includes an exudative phase, a proliferative phase, and a fibrotic phase. Alveolar type II cell hyperplasia occurs after the initial exudative phase, resulting in the formation of resident fibroblasts and extracellular matrix. As disease progresses to the fibrotic phase, extensive basement membrane damage and inadequate or delayed reepithelialization can lead to the development of interstitial and intraalveolar fibrosis, which often requires prolonged mechanical ventilator support [2].

The typical histological hallmark of ARDS is diffuse alveolar damage (DAD) manifesting as hyaline membrane formation, lung edema, inflammation, and hemorrhage [3]. Roughly only half of the patients diagnosed with ARDS based on current criteria present evidence of DAD upon open lung biopsy (OLB) or autopsy [4-7]. Note however that not all ARDS patients progress to the fibrotic phase, as any imbalance 
in profibrotic or antifibrotic mediators could affect progression to the fibroproliferative phase [8]. In patients with ARDS, pulmonary fibrosis often leads to prolonged mechanical ventilation and poor clinical outcomes $[9,10]$.

Idiopathic pulmonary fibrosis (IPF) is a chronic, progressive fibrotic interstitial pneumonia with unknown etiology and poor prognosis. It is characterized by deterioration of the lung parenchyma structure and respiratory function [11]. Acute exacerbation of idiopathic pulmonary fibrosis (AE-IPF) has been defined as an acute, clinically significant respiratory deterioration of unidentifiable cause, commonly leading to acute hypoxemia respiratory failure requiring mechanical ventilation [12]. The typical histological features of AE-IPF are DAD and/or organizing pneumonia superimposed on usual interstitial pneumonia (UIP) pattern (i.e., acute lung injury occurring in an IPF/UIP lung), such that it shares many of the pathological features of ARDS [13, 14].

A lung-protective ventilation strategy remains the cornerstone of treatment for ARDS patients and has been positively correlated with improved survival [2]; however, it brings with it the risk of ventilatorinduced lung injury (VILI) and subsequent lung fibrosis [15]. Researchers have yet to establish optimal ventilator settings for patients with pulmonary fibrosis. Our objective in this study was to examine the association between serial changes in ventilator settings and histological fibrosis in ARDS patients with DAD based on OLB and compare clinical outcomes between fibrosis and non-fibrosis groups.

\section{Methods}

\section{Study design and patients}

This retrospective study was based on analysis of all ARDS patients who underwent OLB at Chang Gung Memorial Hospital (CGMH) in Taiwan between March 2006 and December 2019. CGMH is a tertiary care referral center with a 3700-bed general ward and 278-bed adult intensive care unit (ICU). The exclusion criteria included age of $<20$ years and histological findings not indicative of DAD.

At our institution, the decision to perform OLB was made by the treating intensivist in cases where the etiology of ARDS was unknown and the patient presented rapid pulmonary infiltration following a complete microbiologic examination including bronchoalveolar lavage. Surgical procedures were performed by a chest surgeon in an operating room or at bedside in the ICU. Informed consent was obtained from the family prior to OLB. Under general anesthesia and mechanical ventilator support with adequate oxygenation, video-assisted thoracoscopic surgery or a 5-cm thoracotomy was used to secure the origins using an endoscopic stapler-cutter. The biopsy site was a new or progressive lesion identified via high resolution computed tomography (HRCT) or chest X-ray. Every tissue specimen was examined by pathologists. The local Institutional Review Board for Human Research approved this study (CGMH IRB No. 202000760A3 and 202100595A3), and the need for informed consent was waived due to the retrospective nature of the study.

\section{Definitions}


ARDS was defined in accordance with the Berlin criteria [7]. Dynamic driving pressure $(\Delta P)$ was calculated as peak inspiratory pressure (Ppeak) minus positive end-expiratory pressure (PEEP) [16]. Mechanical power (MP) was calculated using the following equation [17, 18]: MP $($ Joules $/$ minutes $)(\mathrm{J} / \mathrm{min})=0.098 \times$ tidal volume $\left(\mathrm{V}_{\mathrm{T}}\right) \times$ respiratory rate $(\mathrm{RR}) \times($ Ppeak $-1 / 2 \times \Delta \mathrm{P})$.

Ppeak is equivalent to plateau pressure in pressure-controlled ventilation, and Ppeak was used as a surrogate for plateau pressure to calculate MP if not specified [17]. Ventilator-free days was defined as the number of days between day 1 and day 28 or day 90 in which the patient breathed without assistance for at least 48 consecutive hours. Patients who did not survive to 28 days or 90 days were assigned zero ventilator-free days. Patients were stratified into the non-fibrosis group (DAD with exudative or proliferative phase) or the fibrosis group (DAD with fibrotic phase or chronic feature of honeycomb fibrosis).

\section{Data collections}

Demographic data, comorbidities, and the etiologies of ARDS were recorded from hospital charts. The dates of hospital and ICU admission, mechanical ventilator initiation and liberation, date of ARDS diagnosis and OLB, ICU and hospital discharge, and time of death were collected. Arterial blood gas and mechanical ventilator settings parameters were recorded at approximately 10 a.m. daily after ARDS onset.

\section{Histological diagnosis}

Based on analysis of hematoxylin- and eosin-stained lung tissue slices, a diagnosis of DAD (with or without fibrosis) was made independently by at least two pathologists who were blinded to the patients' clinical information. Any discrepancies were discussed by the pathologists with the aim of reaching a final consensus as to histological diagnosis. The presence of DAD indicated hyaline membrane formation, intra-alveolar edema, alveolar type I cell necrosis, alveolar type II cell proliferation, the interstitial proliferation of myofibroblasts and fibroblasts, or organizing interstitial fibrosis [3]. AEIPF represents DAD and/or organizing pneumonia superimposed on UIP pattern [13, 14]. We defined fibrosis as the manifestation of collagenous fibrosis or chronic appearance of microcystic honeycombing, or both [19].

\section{Statistical analysis}

Continuous variables are presented as mean \pm standard deviation or median (interquartile range), and categorical variables are reported as numbers (percentages). A student's $t$-test or the Mann-Whitney $U$ test was used to compare continuous variables among groups. Categorical variables were tested using the chi-square test for equal proportions or the Fisher's exact test. Risk factors associated with histological fibrosis at OLB day were initially analyzed using univariate analysis, followed by a multivariable logistic regression model with stepwise selection. The results are presented using odds ratio and $95 \%$ confidence 
intervals (Cl). All statistical analysis was performed using SPSS 26.0 statistical software, and statistical significance was considered when a two-sided $p$ value is less than 0.05 .

\section{Results}

\section{Study populations}

This study identified 89 ARDS patients who underwent OLB within the study period and screened for inclusion and exclusion criteria. All ARDS patients were deeply sedated and paralyzed during the initial phase, including the day of OLB, and most cases received pressure-controlled ventilation until attempts at weaning from mechanical ventilator. After excluding 21 patients who did not fulfill the histological DAD, 68 patients with histological DAD were analyzed (Fig. 1). Based on histological findings, 56 patients were assigned to the non-fibrosis group, and 12 patients were assigned to the fibrosis group. In the fibrosis group, 7 patients had DAD with fibrotic phase and 5 patients presenting the AE-IPF.

\section{Histological findings}

Compared to normal lung tissue (Fig. 2A), DAD in the initial stage was characterized by the formation of hyaline membrane (Fig. 2B) and in later stage by interstitial fibrosis (Fig. 2C). The term honeycomb fibrosis refers to the typical appearance of cysts in scarred lung tissue (i.e., UIP pattern) (Fig. 2D). Organizing pneumonia was deemed indicative of acute exacerbation of UIP (Fig. 2E).

\section{Baseline characteristics and clinical variables: non-fibrosis and fibrosis groups}

As shown in Table 1, we observed no significant difference between non-fibrosis and fibrosis groups in terms of age, gender, body weight, body mass index, or comorbidities. In both groups, most of the ARDS cases were attributable to pulmonary causes $(n=58,85 \%)$. The median interval between ARDS diagnosis and biopsy was significantly longer in the fibrosis group than the non-fibrosis group (18 [9-30] vs. 8 [512] days, $p=0.024)$. In terms of ventilator settings at the time of ARDS diagnosis, MP, Ppeak, and dynamic $\Delta \mathrm{P}$ levels were significantly higher in the fibrosis group than in the non-fibrosis group (all $p<$ $0.05)$. On the day of biopsy, Ppeak and dynamic $\Delta P$ values remained significantly higher in the fibrosis group than in the non-fibrosis group, and dynamic compliance was significantly lower (all $p<$ 0.05).

The overall hospital mortality rate was $59 \%$, and the mortality was relatively higher in the fibrosis group than in the non-fibrosis group ( $67 \%$ vs. $57 \%, p=0.748)$. The duration of mechanical ventilation, length of ICU stay, and length of hospital stay were significantly higher in the fibrosis group than in the non-fibrosis group (all $p<0.05$ ).

\section{Baseline characteristics and clinical variables: DAD with fibrotic phase and AE-IPF groups}

As shown in Table 2, the patients in the AE-IPF group was older than those in the DAD with fibrotic phase group. There were no significant differences between the two groups in terms of age, gender, body weight, 
body mass index, or comorbidities. In terms of ventilator settings at ARDS diagnosis, we observed no significant differences between the two groups. The median interval between ARDS diagnosis and biopsy was longer in the DAD with fibrotic phase group than the AE-IPF group (28 [17-53] vs. 7 [421] days). On the day of biopsy, patients in the AE-IPF group received higher $M P$, higher $V_{T}$, higher Ppeak, higher mean airway pressure, higher dynamic $\Delta P$, and higher RR than did patients in the DAD with fibrotic phase group, although the difference did not reach the level of significance (Table 2 and Fig. 3). Dynamic compliance was similar between the two groups on the day of biopsy.

\section{Clinical outcomes: DAD with fibrotic phase and AE-IPF}

As shown in Table 3 and Fig. 4, 90-d hospital mortality was higher in the AE-IPF group than in the DAD with fibrotic phase group ( $80 \%$ vs. $57 \%, p=0.242)$. The duration of mechanical ventilation, length of ICU stay, and length of hospital stay were higher in the DAD with fibrotic phase group than the AE-IPF group. The number of ventilator-free days at day 90 , and ICU-free days at day 60 were also higher in the DAD with fibrotic phase group than the AE-IPF group.

\section{Factors associated with histological fibrosis at OLB}

After adjusting for significant confounding variables, a multivariable logistic regression model revealed that patients who had higher MP at ARDS diagnosis and had longer ARDS duration before biopsy were significantly associated with histological fibrosis at OLB (odds ratio 1.493 [95\% Cl 1.014-2.200], $p=$ 0.042 and odds ratio 1.160 [95\% $\mathrm{Cl} 1.052-1.278], p=0.003$, respectively) (Table 4).

\section{Discussion}

The primary insight in this research was the fact that ARDS patients with histological DAD and fibrosis based on OLB received significantly higher airway pressures, and had significantly longer duration of mechanical ventilator, longer length of ICU and hospital stay. Hospital mortality was higher in the fibrosis group than in the non-fibrosis group. In the fibrosis group, patients with AE-IPF received higher ventilator load and had higher hospital mortality than those in the DAD with fibrotic phase group.

DAD is the pathological hallmark of ARDS. In the current study, we enrolled only ARDS patients with histological evidence of DAD using data from OLB, unlike previous studies that included ARDS patients with and without DAD using data from OLB or autopsy $[6,20,21]$. The pathogenesis of ARDS proceeds through an exudative phase (for roughly the first week after ARDS onset), proliferative phase (between the first- and third-weeks following ARDS onset), and fibrotic phase (beyond 3 or 4 weeks after ARDS onset) $[2,22]$. In the current study, the median duration from ARDS diagnosis to OLB was 28 days in the DAD with fibrotic phase group. Some of the patients had progressed to the DAD with fibrotic phase within 3 weeks of ARDS onset. This indicates that the diagnosis of ARDS depends on clinical criteria, and that histological fibrosis may begin before all the criteria for clinical diagnosis of ARDS are met [19]. In a multivariable logistic regression model, longer ARDS duration before biopsy were independently associated with histological fibrosis at OLB. 
The causes of pulmonary fibrosis during ARDS progression are multifactorial (e.g., inflammation and VILI) $[9,15]$. The course of lung fibrosis can be traced to persistent injury and repair in response to mechanical strain and stress on epithelial cell resulting from volutrauma and atelectrauma [23]. MP refers to the energy delivered by a ventilator to the respiratory system per unit of time, as determined by volume, pressure, flow, and RR. Researchers have established that MP is of higher predictive value than individual ventilator parameters in assessing the risk of VILI $[17,18]$. Excessive MP has been shown to promote VILI and appears to be strongly correlated with histology (DAD score) and the expression of interleukin-6, a marker of inflammation [24]. Driving pressure is inversely proportional to lung compliance and aerated remaining functional lung size and has been linked to mortality in ARDS patients $[16,25]$.

There were few studies to examine the correlation between serial changes in ventilator settings and the development of histological fibrosis in ARDS patients. Serial changes of ventilator settings may reflect the severity of nonresolving lung damage. In the current study, the MP and airway pressures (Ppeak and dynamic $\Delta \mathrm{P}$ ) received by the fibrosis group were significantly higher than those received by the nonfibrosis group at ARDS diagnosis. Thus, it is likely that the formation of lung fibrosis can be attributed at least in part to energy load (i.e., MP). In a multivariable logistic regression model, higher MP at ARDS diagnosis was independently associated with histological fibrosis at OLB. Compliance of the respiratory system was associated with the severity of lung injury, duration of ARDS, extent of lung fibrosis, and clinical outcomes [19]. Dynamic compliance in the fibrosis group was significantly lower and dropped more rapidly than in the non-fibrosis group at biopsy day. This may be due to the presence of fibrosis and a longer interval between ARDS diagnosis and OLB in the fibrosis group.

IPF is a form of chronic fibrosing interstitial pneumonia characterized by progressive decline in lung function with radiological and/or histopathological indications of UIP [11, 26]. Note however that UIP is not synonymous with IPF. The UIP pattern of fibrosis has also been linked to other conditions, such as connective tissue disease (mostly rheumatoid arthritis), drug toxicity, chronic hypersensitivity pneumonitis, asbestosis, and Hermansky-Pudlak syndrome [26, 27]. AE-IPF and ARDS are quite similar in terms of DAD, lung inflammation, and respiratory mechanics. AE-IPF can lead to severe acute hypoxemic respiratory failure requiring mechanical ventilator support. Patients with AE-IPF face a higher risk of mortality (may reach to 95\%) [14], which may be related to the fact that IPF patients tend to be older. In our study, five of the twelve patients in the fibrosis group presented histological findings indicative of UIP, and none of those patients presented with connective tissue disease, drug toxicity, or asbestosis. A pathologist and radiologist agreed that the cause of respiratory failure in that group was primarily AE-IPF. Patients in the AE-IPF group were older than those in the DAD with fibrotic phase group (mean age 73.6 vs. 59.3 years), and the mortality was higher than DAD with fibrotic phase group ( $80 \%$ vs $57 \%$ ).

Impaired lung mechanics due to structural, biochemical, and anatomical aberrations render fibrotic lungs susceptibility to VILI [28]. There is at present no solid evidence indicating the optimal ventilator settings for fibrotic lungs, including AE-IPF; some concepts can derive from the evidence regarding ARDS because both share some common features. A "lung resting strategy" to avoid high PEEP during expiration and 
thereby prevent further lung injury has been posited as an alternative to the "open lung approach" (for ARDS cases presenting only DAD) for patients with pulmonary fibrosis and UIP, due to the fact that the presence of fibrotic tissue renders the lung structure highly fragile and prone to VILI (i.e., "squishy ball lung" concept) [28]. In our study, patients in the DAD with fibrotic phase and AE-IPF groups received similar ventilator settings except for $\mathrm{V}_{\mathrm{T}}$ at ARDS diagnosis. However, patients in the AE-IPF group received higher energy load (i.e., MP), higher $\mathrm{V}_{\mathrm{T}}$, and higher airway pressures than in the $D A D$ with fibrotic phase group at biopsy day. It indicated that intensivists may not recognize the disease status well and apply lung-protective ventilation at ARDS onset promptly; however, as disease progression, patients in the AEIPF group received higher ventilator load than those in the DAD with fibrotic phase group at biopsy day due to underlying chronic fibrotic lungs, which contributed to higher risk of VILI.

The strength of our study was that we investigated pulmonary fibrosis based on histological fibrosis from OLB. Previous studies examining the effect of pulmonary fibrosis on clinical outcomes in ARDS patients reported a link between HRCT scores indicative of fibroproliferative changes and clinical outcomes/mortality $[10,29]$. Nonetheless, thin-section CT scanning, including inspiratory, expiratory, and prone sequences, is the most important tool by which to evaluate pulmonary fibrosis. Serial images and quantitative estimates are also essential to differentiating fibrosis progression [27]. Overall, imaging alone cannot be relied upon to confirm destruction of the lung parenchyma, delineate active fibroproliferation, or degree of lung fibrosis [8]. At present, there is no definitive biomarker for DAD, and HRCT findings are insufficient to differentiate DAD from DAD with organizing pneumonia, which was indicative of acute exacerbation of UIP [30]. The only way to confirm the presence of DAD is to obtain lung tissues via OLB or autopsy [20]. Unfortunately, cases that end in autopsy are very likely more severe than live cases, and autopsy series are unable to differentiate clinical outcomes or effects on mortality $[19,20]$. In the current study, we investigated the effect of pulmonary fibrosis on clinical outcomes by enrolling ARDS patients with histological DAD and fibrosis who had undergone OLB.

This retrospective study was hindered by a number of limitations. First, all patients were from a single tertiary care referral center over a long enrollment period. Furthermore, we focused only cases of ARDS that had undergone OLB who fulfilled the histological DAD with fibrosis (i.e., fibrotic phase or AE-IPF) or not, which limited the number of recruited patients. Note that we opted not to exclude the five patients with chronic fibrosis (i.e., UIP pattern) from the fibrosis group $(n=12)$, similar to that of a previous ARDS study based on autopsies in which half of the fibrosis group (15 of 30 patients) also presented with chronic microcystic honeycombing [19]. Besides, we further divided the fibrosis group into the DAD with fibrotic phase or the AE-IPF group and compared clinical outcomes. Second, the causes of pulmonary fibrosis are complex and multifactorial, and the exact causal relationship between mechanical ventilation and pulmonary fibrosis was difficult to determine due to the retrospective nature of the study. Third, compliance with lung-protective ventilation tends to drop in real-world clinical practice over the long study period from 2006 to 2019. The ARDS patients included in this study received relatively high $\mathrm{V}_{T}$, which may have influenced clinical outcomes. Finally, corticosteroids have anti-inflammatory and antifibrosis 
effects; however, we opted not to address the use of steroid therapy, due to a lack of evidence pertaining to the benefits of steroid treatment for persistent ARDS and fibrotic lungs.

\section{Conclusions}

Our findings revealed that ARDS patients with histological DAD and fibrosis received significantly higher airway pressures, underwent mechanical ventilation for a longer duration and remained in the ICU and hospital for a longer period. Hospital mortality was higher in the fibrosis group than in the non-fibrosis group. In the fibrosis group, patients with AE-IPF received higher ventilator load and faced higher mortality than those in DAD with fibrotic phase.

Implementing optimal ventilator settings as early as possible may be necessary to reduce the risk of VILI and pulmonary fibrosis. Further large-scale studies will be required to identify the mechanisms by which mechanical ventilation induces pulmonary fibrosis, and define safety standards aimed at minimizing the risk of VILI and fibrosis.

\section{Abbreviations}

ARDS: acute respiratory distress syndrome; AE-IPF: acute exacerbation of idiopathic pulmonary fibrosis; Cl: confidence interval; ICU: intensive care unit; IPF: idiopathic pulmonary fibrosis; MP: mechanical power; OLB: open lung biopsy; PBW: predicted body weight; PEEP: positive end-expiratory pressure; $\mathrm{V}_{\mathrm{T}}$ : tidal volume; Ppeak: peak inspiratory pressure; $\triangle \mathrm{P}$ : driving pressure; RR: respiratory rate; UIP: usual interstitial pneumonia; VILI: ventilator-induced lung injury

\section{Declarations}

Acknowledgments: The authors would like to express their appreciation to the patients and staff in the ICUs at Chang Gung Memorial Hospital. We thank Mr. Yu-Jr Lin in the Research Services Center for Health Information, Chang Gung University, for validating and confirming all statistics in this study.

\section{Authors' contributions}

HHL and LCC conceived and designed the research; HHL drafted the manuscript;

LCC edited and revised the manuscript. CWW interpreted the pathology and prepared the figures. $\mathrm{CHC}$, $\mathrm{CCH}$, and $\mathrm{HSH}$ interpreted the results from a clinical perspective. HHL and LCC organized the figures. All authors interpreted the results and approved the final version of the manuscript prior to submission.

\section{Funding}

This research was funded by grants CMRPG3K1151 and CMRPG3L0821 from Chang Gung Memorial Hospital. 
Availability of data and materials

The datasets used and/or analyzed during the current study are available from the corresponding author on reasonable request.

\section{Ethics approval and consent to participate}

The study was conducted in accordance with the guidelines of the Declaration of Helsinki and approved by the Institutional Review Board of CGMH IRB No. 202000760A3 and 202100595A3.

\section{Consent for publication}

Not applicable.

\section{Competing interests}

The authors declare that they have no competing interests.

\section{References}

1. Bellani G, Laffey JG, Pham T, Fan E, Brochard L, Esteban A, et al. Epidemiology, Patterns of Care, and Mortality for Patients With Acute Respiratory Distress Syndrome in Intensive Care Units in 50 Countries. JAMA. 2016;315:788-800.

2. Thompson, B.T., Chambers, R.C., Liu, K.D. Acute Respiratory Distress Syndrome. N Engl J Med 2017;377:562-72.

3. Katzenstein, A.L., Bloor, C.M., Leibow, A.A. Diffuse alveolar damage-the role of oxygen, shock, and related factors. A review. Am J Pathol 1976;85: 209-28.

4. Guerin C, Bayle F, Leray V, Debord S, Stoian A, Yonis H, et al. Open lung biopsy in nonresolving ARDS frequently identifies diffuse alveolar damage regardless of the severity stage and may have implications for patient management. Intensive Care Med. 2015;41:222-30.

5. Kao KC, Hu HC, Chang CH, Hung CY, Chiu LC, Li SH, et al. Diffuse alveolar damage associated mortality in selected acute respiratory distress syndrome patients with open lung biopsy. Crit Care. 2015;19:228.

6. Lorente JA, Cardinal-Fernández P, Muñoz D, Frutos-Vivar F, Thille AW, Jaramillo C, et al. Acute respiratory distress syndrome in patients with and without diffuse alveolar damage: an autopsy study. Intensive Care Med. 2015;41:1921-30.

7. ARDS Definition Task Force, Ranieri VM, Rubenfeld GD, Thompson BT, Ferguson ND, Caldwell E, et al Acute respiratory distress syndrome: the Berlin Definition. JAMA. 2012;307:2526-33.

8. Burnham EL, Janssen WJ, Riches DW, Moss M, Downey GP. The fibroproliferative response in acute respiratory distress syndrome: mechanisms and clinical significance. Eur Respir J. 2014;43:276-85. 
9. Cabrera-Benitez NE, Laffey JG, Parotto M, Spieth PM, Villar J, Zhang H, et al. Mechanical ventilationassociated lung fibrosis in acute respiratory distress syndrome: a significant contributor to poor outcome. Anesthesiology. 2014;121:189-98.

10. Ichikado K, Muranaka H, Gushima Y, Kotani T, Nader HM, Fujimoto K, et al. Fibroproliferative changes on high-resolution $\mathrm{CT}$ in the acute respiratory distress syndrome predict mortality and ventilator dependency: a prospective observational cohort study. BMJ Open. 2012;2:e000545.

11. Raghu G, Remy-Jardin M, Myers JL, Richeldi L, Ryerson CJ, Lederer DJ, et al. Diagnosis of Idiopathic Pulmonary Fibrosis. An Official ATS/ERS/JRS/ALAT Clinical Practice Guideline. Am J Respir Crit Care Med. 2018;198:e44-e68.

12. Collard HR, Ryerson CJ, Corte TJ, Jenkins G, Kondoh Y, Lederer DJ, et al. Acute Exacerbation of Idiopathic Pulmonary Fibrosis. An International Working Group Report. Am J Respir Crit Care Med. 2016;194:265-75.

13. Kim DS, Park JH, Park BK, Lee JS, Nicholson AG, Colby T. Acute exacerbation of idiopathic pulmonary fibrosis: frequency and clinical features. Eur Respir J. 2006;27:143-50.

14. Marchioni A, Tonelli R, Ball L, Fantini R, Castaniere I, Cerri S, et al. Acute exacerbation of idiopathic pulmonary fibrosis: lessons learned from acute respiratory distress syndrome? Crit Care. 2018;22:80.

15. Slutsky, A.S., Ranieri, V.M. Ventilator-induced lung injury. N Engl J Med 2013;369:2126-36.

16. Chiu LC, Hu HC, Hung CY, Chang $\mathrm{CH}$, Tsai FC, Yang CT, et al. Dynamic driving pressure associated mortality in acute respiratory distress syndrome with extracorporeal membrane oxygenation. Ann Intensive Care. 2017;7:12.

17. Chiu LC, Lin SW, Chuang LP, Li HH, Liu PH, Tsai FC, et al. Mechanical power during extracorporeal membrane oxygenation and hospital mortality in patients with acute respiratory distress syndrome. Crit Care. 2021;25:13.

18. Gattinoni L, Tonetti T, Cressoni M, Cadringher P, Herrmann P, Moerer O, et al. Ventilator-related causes of lung injury: the mechanical power. Intensive Care Med 2016;42:1567-75.

19. Thille AW, Esteban A, Fernández-Segoviano P, Rodriguez JM, Aramburu JA, Vargas-Errázuriz P, et al. Chronology of histological lesions in acute respiratory distress syndrome with diffuse alveolar damage: a prospective cohort study of clinical autopsies. Lancet Respir Med. 2013;1:395-401.

20. Cardinal-Fernández P, Bajwa EK, Dominguez-Calvo A, Menéndez JM, Papazian L, Thompson BT. The Presence of Diffuse Alveolar Damage on Open Lung Biopsy Is Associated With Mortality in Patients With Acute Respiratory Distress Syndrome: A Systematic Review and Meta-Analysis. Chest. 2016;149:1155-64.

21. Thille AW, Esteban A, Fernández-Segoviano P, Rodriguez JM, Aramburu JA, Peñuelas O, et al. Comparison of the Berlin definition for acute respiratory distress syndrome with autopsy. Am $J$ Respir Crit Care Med. 2013;187:761-7.

22. Tomashefski, J.F., Jr. Pulmonary pathology of acute respiratory distress syndrome. Clin Chest Med 2000;21:435-466. 
23. Albert RK, Smith B, Perlman CE, Schwartz DA. Is Progression of Pulmonary Fibrosis due to Ventilation-induced Lung Injury? Am J Respir Crit Care Med. 2019;200:140-151.

24. Santos RS, Maia LA, Oliveira MV, Santos CL, Moraes L, Pinto EF, et al. Biologic Impact of Mechanical Power at High and Low Tidal Volumes in Experimental Mild Acute Respiratory Distress Syndrome. Anesthesiology. 2018;128:1193-1206.

25. Amato MB, Meade MO, Slutsky AS, Brochard L, Costa EL, Schoenfeld DA, et al. Driving pressure and survival in the acute respiratory distress syndrome. N Engl J Med. 2015;372:747-55.

26. Wuyts WA, Cavazza A, Rossi G, Bonella F, Sverzellati N, Spagnolo P. Differential diagnosis of usual interstitial pneumonia: when is it truly idiopathic? Eur Respir Rev. 2014;23:308-19.

27. Hobbs S, Chung JH, Leb J, Kaproth-Joslin K, Lynch DA. Practical Imaging Interpretation in Patients Suspected of Having Idiopathic Pulmonary Fibrosis: Official Recommendations from the Radiology Working Group of the Pulmonary Fibrosis Foundation. Radiol Cardiothorac Imaging. 2021;3:e200279.

28. Marchioni A, Tonelli R, Rossi G, Spagnolo P, Luppi F, Cerri S, et al. Ventilatory support and mechanical properties of the fibrotic lung acting as a "squishy ball". Ann Intensive Care. 2020;10:13.

29. Kamo T, Tasaka S, Suzuki T, Asakura T, Suzuki S, Yagi K, et al. Prognostic values of the Berlin definition criteria, blood lactate level, and fibroproliferative changes on high-resolution computed tomography in ARDS patients. BMC Pulm Med. 2019;19:37.

30. Chung JH, Kradin RL, Greene RE, Shepard JA, Digumarthy SR. CT predictors of mortality in pathology confirmed ARDS. Eur Radiol. 2011;21:730-7.

\section{Tables}

Table 1 Background characteristics and clinical variables: non-fibrosis and fibrosis on histological findings 


\begin{tabular}{|c|c|c|c|c|}
\hline Characteristics & $\begin{array}{c}\text { All } \\
(n=68)\end{array}$ & $\begin{array}{l}\text { Non-fibrosis } \\
(\mathrm{n}=56)\end{array}$ & $\begin{array}{l}\text { Fibrosis } \\
(\mathrm{n}=12)\end{array}$ & $p$ \\
\hline Age (years) & $60.4 \pm 16$ & $59.4 \pm 14.8$ & $65.3 \pm 20.8$ & 0.255 \\
\hline Male (gender) & $39(57 \%)$ & 31 (55\%) & 8 (67\%) & 0.472 \\
\hline Body weight (kg) & $60.7 \pm 11.7$ & $61.0 \pm 12.3$ & $58.4 \pm 6.1$ & 0.566 \\
\hline Body mass index $\left(\mathrm{kg} / \mathrm{m}^{2}\right)$ & $23.7 \pm 3.8$ & $23.8 \pm 3.9$ & $22.8 \pm 3.3$ & 0.488 \\
\hline \multicolumn{5}{|l|}{ Comorbidities } \\
\hline Diabetes mellitus & $12(18 \%)$ & $9(16 \%)$ & $3(25 \%)$ & 0.432 \\
\hline Hypertension & $20(29 \%)$ & $18(32 \%)$ & $2(17 \%)$ & 0.486 \\
\hline Chronic lung diseases & $7(10 \%)$ & $6(11 \%)$ & $1(8 \%)$ & 1.0 \\
\hline Immunocompromised & $18(27 \%)$ & $16(29 \%)$ & $2(17 \%)$ & 0.494 \\
\hline \multicolumn{5}{|l|}{ ARDS etiologies } \\
\hline Pulmonary causes & $58(85 \%)$ & $47(84 \%)$ & $11(92 \%)$ & 0.678 \\
\hline Extrapulmonary causes & $10(15 \%)$ & $9(16 \%)$ & $1(8 \%)$ & 0.678 \\
\hline $\begin{array}{l}\mathrm{PaO}_{2} / \mathrm{FiO}_{2} \text { at day of ARDS diagnosis } \\
(\mathrm{mmHg})\end{array}$ & $\begin{array}{l}135(61- \\
204)\end{array}$ & $\begin{array}{l}136(61- \\
213)\end{array}$ & $\begin{array}{l}118(58- \\
187)\end{array}$ & 0.613 \\
\hline \multicolumn{5}{|c|}{ Ventilator settings at day of ARDS diagnosis } \\
\hline Mechanical power (J/min) & $24.6 \pm 9$ & $23.5 \pm 8.2$ & $29.9 \pm 10.8$ & 0.023 \\
\hline Tidal volume (ml/kg PBW) & $8.6 \pm 1.9$ & $8.66 \pm 2$ & $8.42 \pm 1.6$ & 0.764 \\
\hline $\operatorname{PEEP}\left(\mathrm{cm} \mathrm{H}_{2} \mathrm{O}\right)$ & $10.9 \pm 2.4$ & $11 \pm 2.5$ & $11 \pm 2.1$ & 0.566 \\
\hline Peak inspiratory pressure $\left(\mathrm{cm} \mathrm{H}_{2} \mathrm{O}\right)$ & $31.7 \pm 5.6$ & $31.1 \pm 4.9$ & $36.1 \pm 5.3$ & 0.004 \\
\hline Mean airway pressure $\left(\mathrm{cm} \mathrm{H}_{2} \mathrm{O}\right)$ & $17.6 \pm 3.5$ & $17.5 \pm 3.4$ & $18.5 \pm 4$ & 0.345 \\
\hline Dynamic driving pressure $\left(\mathrm{cm} \mathrm{H}_{2} \mathrm{O}\right)$ & $20.9 \pm 5.2$ & $20.2 \pm 4.4$ & $24 \pm 7.5$ & 0.021 \\
\hline Total respiratory rate (breaths/min) & $24.9 \pm 5$ & $24.3 \pm 4.9$ & $27.3 \pm 5.2$ & 0.088 \\
\hline Dynamic compliance $\left(\mathrm{ml} / \mathrm{cm} \mathrm{H}_{2} \mathrm{O}\right)$ & $24.8 \pm 10.5$ & $24.8 \pm 8.9$ & $24.8 \pm 16.5$ & 0.989 \\
\hline Day from ARDS diagnosis to biopsy & $8(5-14)$ & $8(5-12)$ & $18(9-30)$ & 0.024 \\
\hline $\mathrm{PaO}_{2} / \mathrm{FiO}_{2}$ at biopsy day $(\mathrm{mmHg})$ & $\begin{array}{l}139(104- \\
194)\end{array}$ & $\begin{array}{l}137(103- \\
199)\end{array}$ & $\begin{array}{l}141(98- \\
191)\end{array}$ & 0.867 \\
\hline \multicolumn{5}{|l|}{ Ventilator settings at biopsy day } \\
\hline Mechanical power (J/min) & $23.8 \pm 7.7$ & $23.1 \pm 6.9$ & $27 \pm 10.3$ & 0.12 \\
\hline Tidal volume (ml/kg PBW) & $7.7 \pm 1.8$ & $7.7 \pm 1.7$ & $7.6 \pm 2.3$ & 0.961 \\
\hline $\operatorname{PEEP}\left(\mathrm{cm} \mathrm{H}_{2} \mathrm{O}\right)$ & $12.3 \pm 2.6$ & $12.5 \pm 2.5$ & $11.3 \pm 2.6$ & 0.191 \\
\hline Peak inspiratory pressure $\left(\mathrm{cm} \mathrm{H}_{2} \mathrm{O}\right)$ & $34.4 \pm 6.9$ & $33.6 \pm 6.2$ & $38.2 \pm 9.1$ & 0.037 \\
\hline Mean airway pressure $\left(\mathrm{cm} \mathrm{H}_{2} \mathrm{O}\right)$ & $19.3 \pm 3.8$ & $19.4 \pm 3.6$ & $18.8 \pm 5.1$ & 0.567 \\
\hline Dynamic driving pressure $\left(\mathrm{cm} \mathrm{H}_{2} \mathrm{O}\right)$ & $22.1 \pm 6.8$ & $21.1 \pm 6.0$ & $26.8 \pm 8.2$ & 0.007 \\
\hline Total respiratory rate (breaths/min) & $25.2 \pm 5$ & $24.9 \pm 4.7$ & $26.3 \pm 6.5$ & 0.373 \\
\hline Dynamic compliance $\left(\mathrm{ml} / \mathrm{cm} \mathrm{H}_{2} \mathrm{O}\right)$ & 7.6 & 7.6 & \pm 6.7 & 0.017 \\
\hline Hospital mortality, n (\%) & $40(59 \%)$ & $32(57 \%)$ & $8(67 \%)$ & 0.748 \\
\hline $\begin{array}{l}\text { Duration of mechanical ventilator } \\
\text { (days) }\end{array}$ & $22(15-34)$ & $21(14-32)$ & $35(24-74)$ & 0.028 \\
\hline Length of ICU stay (days) & $27(17-37)$ & $25(16-34)$ & $51(32-80)$ & 0.001 \\
\hline Length of hospital stay (days) & $34(22-56)$ & $31(20-46)$ & $55(32-81)$ & 0.004 \\
\hline Ventilator-free days at day 28 & $0(0-5)$ & $0(0-11)$ & $0(0-0)$ & 0.036 \\
\hline [CU-free days at day 28 & $0(0-7)$ & $0(0-8)$ & $0(0-0)$ & 0.036 \\
\hline [CU-free days at day 60 & $0(0-35)$ & $0(0-40)$ & $0(0-0)$ & 0.008 \\
\hline Hospital-free days at day 90 & $51(29-66)$ & $54(16-34)$ & $25(2-54)$ & 0.01 \\
\hline
\end{tabular}

Data was presented as mean \pm standard deviation, count or median (interquartile range) $A R D S$ acute respiratory distress syndrome, $\mathrm{FiO}_{2}$ fraction of inspired oxygen, ICU intensive care unit, $\mathrm{PaO}_{2}$ partial pressure of oxygen in arterial blood, $P B W$ predicted body weight, $P E E P$ positive end-expiratory pressure 
Table 2 Background characteristics and clinical variables: DAD with fibrotic phase and AEIPF on histological findings

\begin{tabular}{|c|c|c|c|}
\hline Characteristics & $\begin{array}{l}\text { DAD with fibrotic } \\
\text { phase }(\mathrm{n}=7)\end{array}$ & $\begin{array}{c}\text { AE-IPF }(n= \\
5)\end{array}$ & $p$ \\
\hline Age (years) & $59.3 \pm 25.5$ & $73.6 \pm 8.36$ & 0.204 \\
\hline Male (gender) & $5(\overline{71} \%)$ & $3(60 \%)$ & 0.769 \\
\hline Body weight (kg) & $60.5 \pm 8.1$ & $56.4 \pm 3.2$ & 0.379 \\
\hline Body mass index $\left(\mathrm{kg} / \mathrm{m}^{2}\right)$ & $23.4 \pm 4.4$ & $22.1 \pm 1.7$ & 0.657 \\
\hline \multicolumn{4}{|l|}{ Comorbidities } \\
\hline Diabetes mellitus & $1(14 \%)$ & $2(40 \%)$ & 0.31 \\
\hline Hypertension & $1(14 \%)$ & $1(20 \%)$ & 0.793 \\
\hline Chronic lung diseases & $1(14 \%)$ & $0(0 \%)$ & 0.377 \\
\hline Immunocompromised & $2(29 \%)$ & $0(0 \%)$ & 0.19 \\
\hline \multicolumn{4}{|l|}{ ARDS etiologies } \\
\hline Pulmonary causes & $6(86 \%)$ & $5(100 \%)$ & 1.0 \\
\hline Extrapulmonary causes & $1(14 \%)$ & $0(0 \%)$ & 1.0 \\
\hline $\begin{array}{l}\mathrm{PaO}_{2} / \mathrm{FiO} \\
(\mathrm{mmHq})\end{array}$ & $68(39-138)$ & $\begin{array}{l}136(118- \\
176)\end{array}$ & 0.794 \\
\hline \multicolumn{4}{|c|}{ Ventilator settings at day of ARDS diagnosis } \\
\hline Mechanical power (J/min) & $30 \pm 8.7$ & $30 \pm 14$ & 0.987 \\
\hline Tidal volume (ml/kg PBW) & $8.9 \pm 1.9$ & $7.8 \pm 1.1$ & 0.434 \\
\hline $\operatorname{PEEP}\left(\mathrm{cm} \mathrm{H}_{2} \mathrm{O}\right)$ & $10.6 \pm 2.2$ & $10.4 \pm 2.2$ & 0.897 \\
\hline Peak inspiratory pressure $\left(\mathrm{cm} \mathrm{H}_{2} \mathrm{O}\right)$ & $34.6 \pm 5.6$ & $34.4 \pm 5.7$ & 0.971 \\
\hline Mean airway pressure $\left(\mathrm{cm} \mathrm{H}_{2} \mathrm{O}\right)$ & $18.6 \pm 4.1$ & $18.4 \pm 4.4$ & 0.946 \\
\hline Dynamic driving pressure $\left(\mathrm{cm} \mathrm{H}_{2} \mathrm{O}\right)$ & $24 \pm 5.2$ & $24 \pm 10.7$ & 1.0 \\
\hline Total respiratory rate (breaths/min) & $27.1 \pm 4.6$ & $27.6 \pm 6.6$ & 0.889 \\
\hline Dynamic compliance $\left(\mathrm{ml} / \mathrm{cm} \mathrm{H}_{2} \mathrm{O}\right)$ & $21.2(15-24.6)$ & $\begin{array}{l}15.9(14.1- \\
49)\end{array}$ & 0.755 \\
\hline Day from ARDS diagnosis to biopsy & $28(17-53)$ & $7(4-21)$ & 0.242 \\
\hline $\mathrm{PaO}_{2} / \mathrm{FiO}_{2}$ at biopsy day $(\mathrm{mmHg})$ & $168(95-192)$ & $106(89-141)$ & 0.546 \\
\hline \multicolumn{4}{|l|}{ Ventilator settings at biopsy day } \\
\hline Mechanical power $(\mathrm{J} / \mathrm{min})$ & $25.2 \pm 11.4$ & $29.4 \pm 9.2$ & 0.851 \\
\hline Tidal volume (ml/kg PBW) & $6.8 \pm 2.7$ & $8.8 \pm 1.3$ & 0.301 \\
\hline PEEP $\left(\mathrm{cm} \mathrm{H}_{2} \mathrm{O}\right)$ & $11 \pm 3.2$ & $11.8 \pm 1.8$ & 0.394 \\
\hline Peak inspiratory pressure $\left(\mathrm{cm} \mathrm{H}_{2} \mathrm{O}\right)$ & $37.4 \pm 10$ & $39.2 \pm 8.8$ & 0.757 \\
\hline Mean airway pressure $\left(\mathrm{cm} \mathrm{H}_{2} \mathrm{O}\right)$ & $17.7 \pm 5.3$ & $20.4 \pm 4.9$ & 0.628 \\
\hline Dynamic driving pressure $\left(\mathrm{cm} \mathrm{H}_{2} \mathrm{O}\right)$ & $26.4 \pm 8.8$ & $27.4 \pm 8.3$ & 0.663 \\
\hline Total respiratory rate (breaths/min) & $25.3 \pm 3.8$ & $27.8 \pm 9.5$ & 0.537 \\
\hline Dynamic compliance $\left(\mathrm{ml} / \mathrm{cm} \mathrm{H}_{2} \mathrm{O}\right)$ & $15.8(11.7-18.6)$ & $\begin{array}{l}15.2(10.1- \\
27.6)\end{array}$ & 0.876 \\
\hline
\end{tabular}

Data was presented as mean \pm standard deviation, count or median (interquartile range) $A E-I P F$ acute exacerbation of idiopathic pulmonary fibrosis, $A R D S$ acute respiratory distress syndrome, $\mathrm{DAD}$ diffuse alveolar damage, $\mathrm{FiO}_{2}$ fraction of inspired oxygen, $\mathrm{PaO}_{2}$ partial pressure of oxygen in arterial blood, $P B W$ predicted body weight, $P E E P$ positive end-expiratory pressure 
Table 3 Clinical outcomes as a function of DAD with fibrotic phase and AE-IPF on histological findings

\begin{tabular}{lccc}
\hline Outcomes & $\begin{array}{c}\text { DAD with fibrotic phase (n } \\
=7)\end{array}$ & $\begin{array}{c}\text { AE-IPF (n }= \\
\text { 5) }\end{array}$ & $p$ \\
\hline $\begin{array}{l}\text { 90-day hospital mortality, n (\%) } \\
\text { Other outcomes }\end{array}$ & $4(57 \%)$ & $4(80 \%)$ & 0.242 \\
$\begin{array}{l}\text { Duration of mechanical ventilator } \\
\text { (days) }\end{array}$ & $46(22-75)$ & $42(24-66)$ & 0.84 \\
\hline $\begin{array}{l}\text { Length of ICU stay (days) } \\
\text { Length of hospital stay (days) }\end{array}$ & $72(37-81)$ & $48(24-78)$ & 0.319 \\
\hline Ventilator-free days at day 90 & $82(53-93)$ & $57(27-98)$ & 0.364 \\
\hline ICU-free days at day 60 & $14(0-23)$ & $0(0-0)$ & 0.224 \\
\hline
\end{tabular}

Data was presented as count or median (interquartile range). $A E-I P F$ acute exacerbation of idiopathic pulmonary fibrosis, $D A D$ diffuse alveolar damage, $I C U$ intensive care unit

Table 4 Factors associated with histological fibrosis at open lung biopsy using a multivariable logistic regression model.

\begin{tabular}{lcc}
\hline Characteristics & Odds ratio (95\% CI) & $p$ \\
\hline MP at day of ARDS diagnosis (J/min) & $1.493(1.014-2.200)$ & 0.042 \\
ARDS duration before biopsy (days) & $1.160(1.052-1.278)$ & 0.003 \\
\hline
\end{tabular}

$A R D S$ acute respiratory distress syndrome, $C I$ confidence interval, $M P$ mechanical power The multivariable analysis model included continuous variables (age, body weight, body mass index, ventilatory variables at day of ARDS diagnosis, and day from ARDS diagnosis to biopsy) and categorical variables (gender, comorbidities, and ARDS etiologies). For the continuous variables, the odds ratio means that the risk of histological fibrosis at open lung biopsy increases or decreases per unit increase of these variables.

\section{Figures}




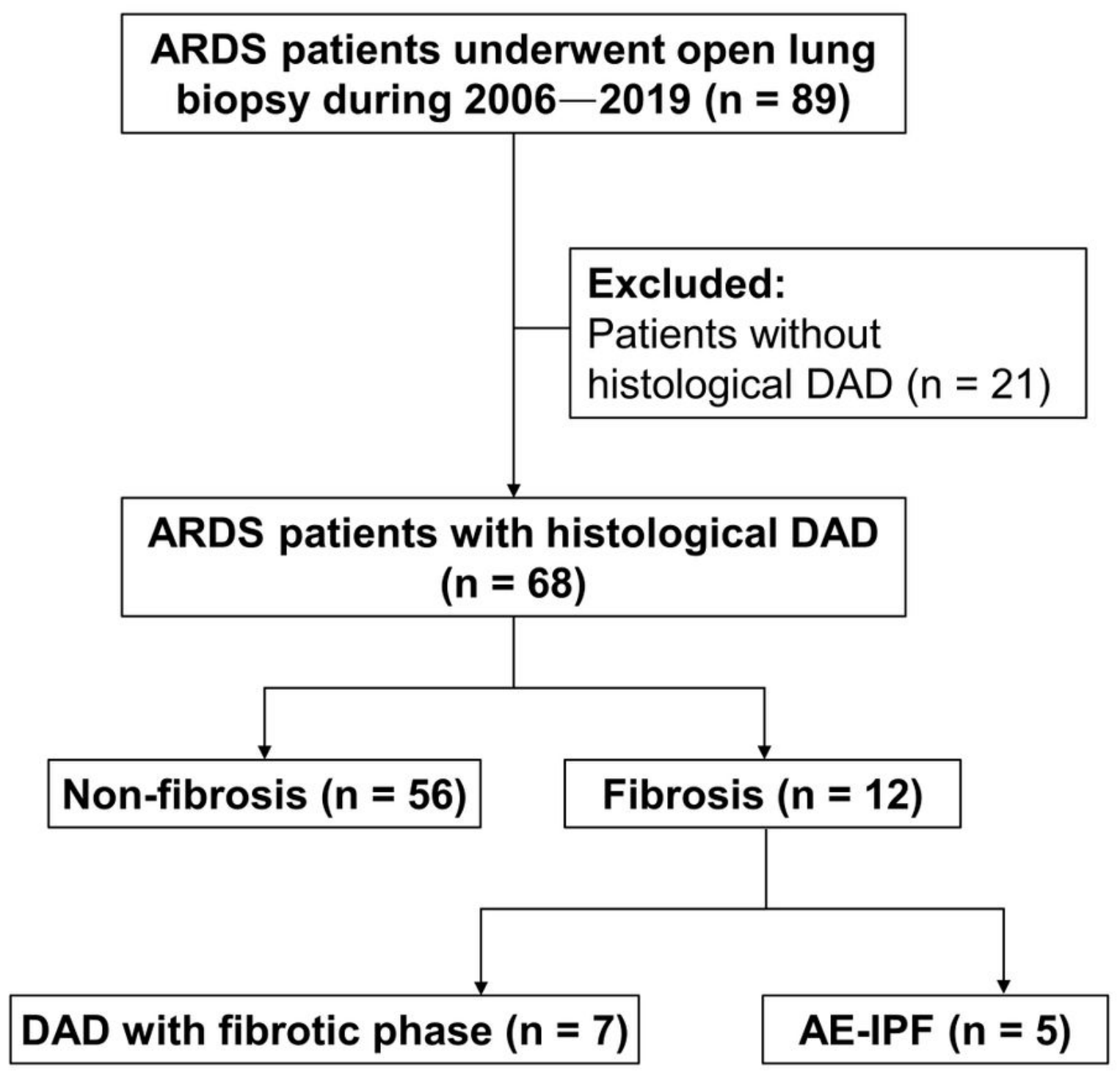

Figure 1

Flow chart of ARDS patients receiving open lung biopsy. ARDS acute respiratory distress syndrome, $D A D$ diffuse alveolar damage, $A E-I P F$ acute exacerbation of idiopathic pulmonary fibrosis 

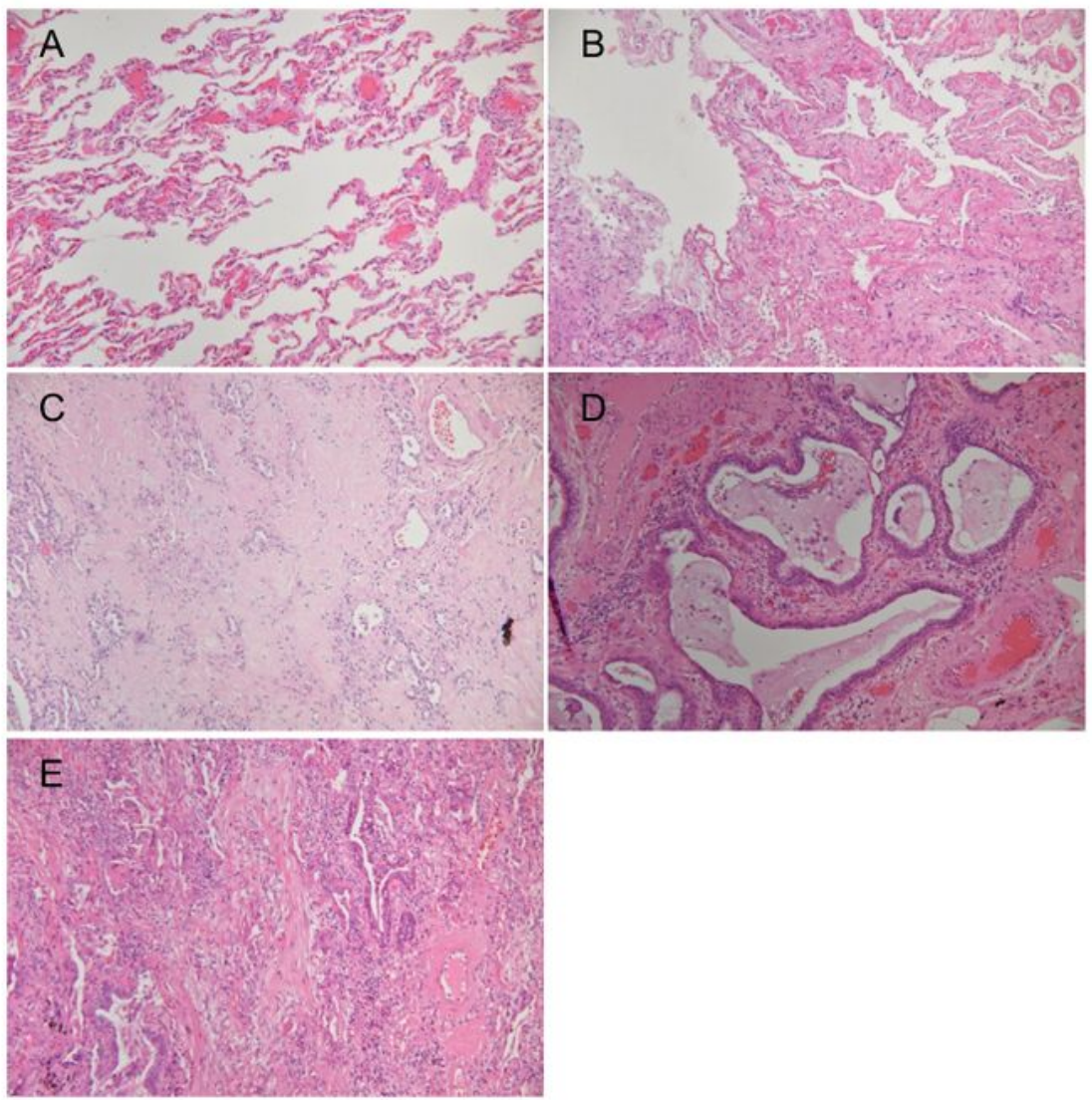

\section{Figure 2}

Human lung tissues samples from enrolled patients stained using hematoxylin and eosin. A Normal lung tissue defined as thin alveolar-capillary membrane with clear alveolar space; B Diffuse alveolar damage in which alveolar surfaces are lined with hyaline membranes; $\mathbf{C}$ Lung tissue showing distinct indications of interstitial fibrosis; D UIP showing honeycomb fibrosis; E Organizing pneumonia indicating acute exacerbation of UIP. 4X magnification. UIP usual interstitial pneumonia 
A

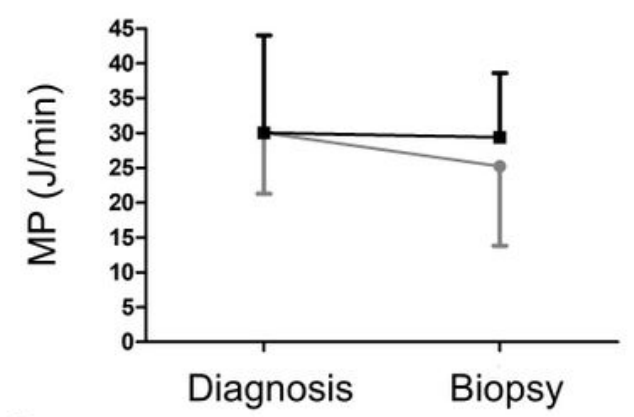

C

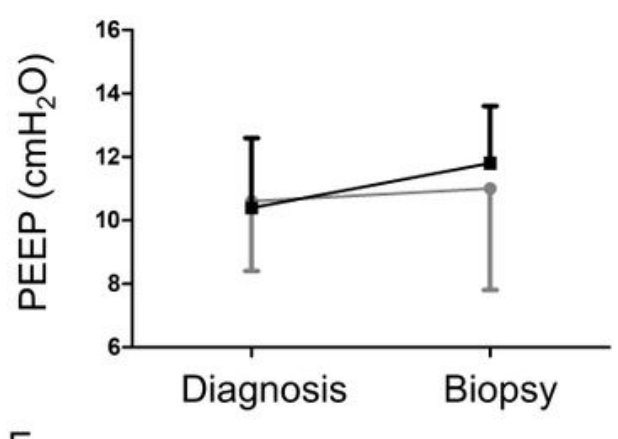

$E$

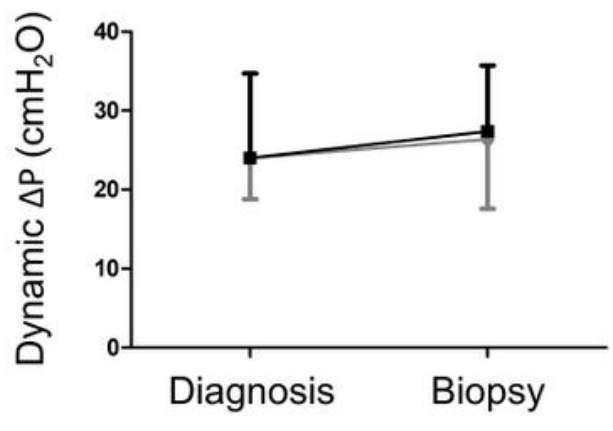

$\rightarrow$ AE-IPF $\rightarrow$ DAD with fibrotic phase
B

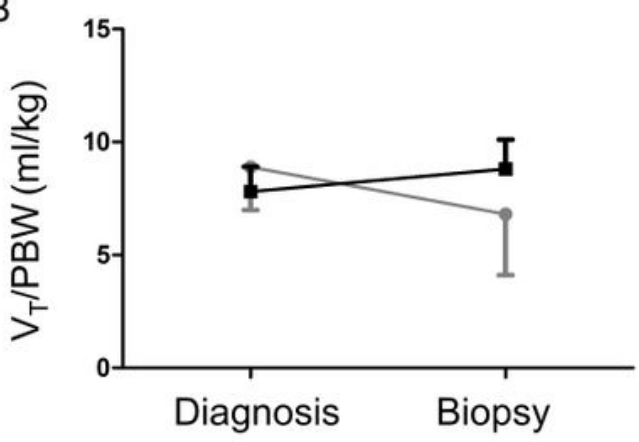

D

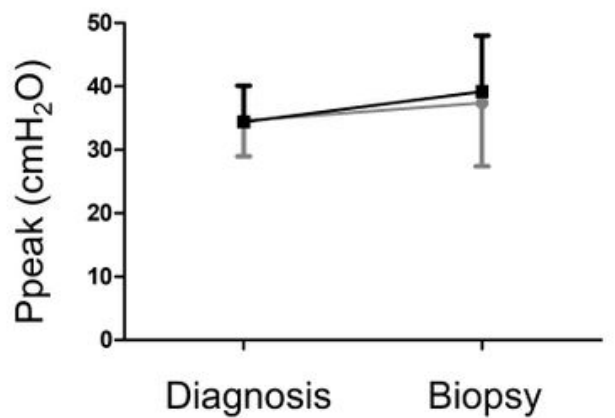

$\mathrm{F}$

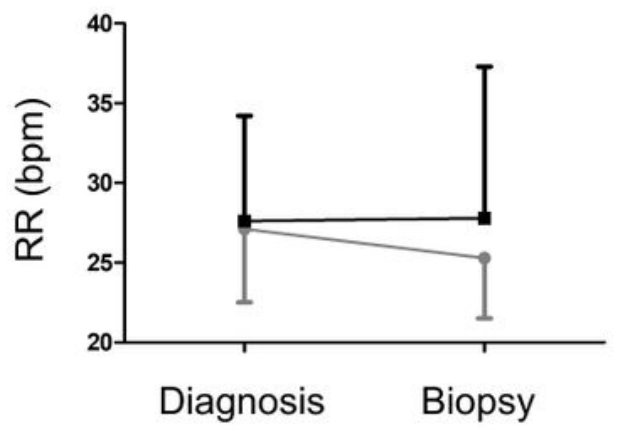

\section{Figure 3}

Serial changes in ventilatory variables between ARDS diagnosis and open lung biopsy of patients in the DAD with fibrotic phase and AE-IPF groups. ARDS acute respiratory distress syndrome, DAD diffuse alveolar damage, $A E-I P F$ acute exacerbation of idiopathic pulmonary fibrosis, $M P$ mechanical power, $V_{T}$ tidal volume, $P B W$ predicted body weight, $P E E P$ positive end-expiratory pressure, $P$ peak peak inspiratory pressure, $\Delta P$ driving pressure, $R R$ respiratory rate, $b p m$ beats per minute 


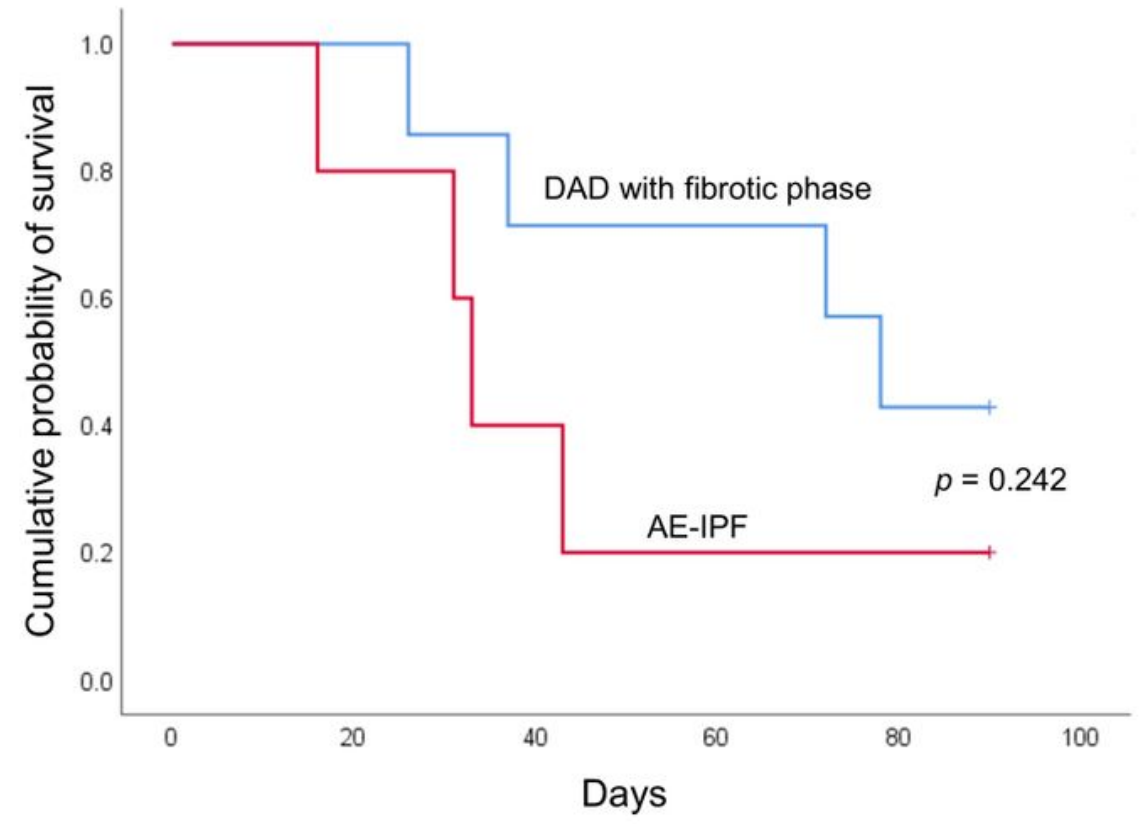

Number at risk

$\begin{array}{llllll}\text { DAD with fibrotic phase } & 7 & 6 & 5 & 5 & 3 \\ \text { AE-IPF } & 5 & 4 & 2 & 1 & 1\end{array}$

\section{Figure 4}

Kaplan-Meier 90-d survival curves of patients with acute respiratory distress syndrome undergoing open lung biopsy, as stratified by DAD with fibrotic phase and AE-IPF. DAD diffuse alveolar damage, $A E-I P F$ acute exacerbation of idiopathic pulmonary fibrosis 